\title{
Parasitóides Associados a Cochonilhas e Aleirodídeos (Sternorrhyncha) de Plantas Cítricas no Estado do Rio de Janeiro
}

\author{
William Costa Rodrigues ${ }^{\circledR}$ \& Paulo Cesar Rodrigues Cassino²
}

1. Universidade Severino Sombra/Entomologistas do Brasil, e-mail: wcrodrigues@ebras.bio.br (Autor para correspondência ${ }^{\varpi}$ ). 2. Universidade Severino Sombra/Universidade Federal Rural do Rio de Janeiro, e-mail: pr.cassino@uol.com.br.

EntomoBrasilis 5 (1): 33-36 (2012)

\begin{abstract}
Resumo. Com o propósito de conhecer os parasitóides de cochonilhas e aleirodídeos, associados às plantas cítricas no Estado do Rio de Janeiro, foram realizadas coletas de folhas contendo os fitoparasitos: Aleurothrixus floccosus (Maskell), Tetraleurodes (=Aleurotrachelus) cruzi (Cassino), Paraleyrodes bondari Peracchi, Coccus viridis (Green), Crysomphalus ficus (Ashmead), Orthezia praelonga (Douglas) e Pinnaspis aspidistrae (Signoret), sendo estas limpas, em seguida procedia-se a eliminação dos organismo que eram objeto de estudo (cada folha permanecia com um única espécie de fitoparasito, para que os parasitóides que surgissem fossem mesmo associados àquela espécie). Após este procedimento as folhas foram incubadas por um período de 25 dias, onde foi observado somente o parasitismo de A. floccosus, P. aspidistrae, C. viridis e T. cruzi, sendo que os parasitóides deste dois últimos, não foram identificados, pois o número de espécimes obtidos foi muito reduzido. Parasitando $A$. floccosus observouse: Arrhenophagus sp., Cales sp., Encarsia sp. (1), Eretmocerus sp., Signiphora sp. (1), Signiphora sp. (2), Signiphora sp. (3) e uma espécie da família Platygasteridae. Parasitando P. aspidistrae observou-se: Arrhenophagus sp., Cales sp., Encarsia sp. (2) e Pteroptrix sp. Algumas espécies estão associadas tanto $A$. floccosus quanto $P$. aspidistrae. Através dos resultados verificou-se que o parasitismo de Arrhenophagus sp. e a espécie da família Platygasteridae em A. floccosus e o parasitismo de Arrhenophagus sp. e Pteroptrix sp. em P. aspidistrae, são novos registros de ocorrência para o Estado do Rio de Janeiro.
\end{abstract}

Palavras-Chave: Citros; Controle biológico; inimigos naturais; Hymenoptera; Parasitismo.

\section{Parasitoids Associated to Scales and Whiteflies (Sternorrhyncha) of Citrus Plants in Rio de Janeiro State}

Abstract. With the purpose of knowing parasitids of scales and whiteflies, associates to the citrus plants in Rio de Janeiro State, they were accomplished leaves collected contend fitoparasits: Aleurothrixus floccosus (Maskell), Tetraleurodes (=Aleurotrachelus) cruzi (Cassino), Paraleyrodes bondari Peracchi, Coccus viridis (Green), Crysomphalus ficus (Ashmead), Orthezia praelonga (Douglas) and Pinnaspis aspidistrae (Signoret), being this ones clean, it soon after proceded the organism elimination that was study object (each leaf remained with an only species of fitoparasite, so that parasitoids that had arisen had gone same associate to that species). After this procedure the leaves were incubate for a period of 25 days, when it was observed only parasitism in A. floccosus, $P$. aspidistrae, $C$. viridis and T. cruzi, and parasitoids of this the latter, not identified because the number specimens was very reduced. It was parasiting A. floccosus it observed: Arrhenophagus sp., Cales sp., Encarsia sp. (1), Eretmocerus sp., Signiphora sp. (1), Signiphora sp. (2), Signiphora sp. (3) and a species of the family Platygasteridae. Parasiting P. aspidistrae it was observed: Arrhenophagus sp., Cales sp., Encarsia sp. (2) and Pteroptrix sp. Some species are associated either to A. floccosus or to P. aspidistrae. By the results it was verified that Arrhenophagus sp. the parasitism and the species of the family Platygasteridae in A. floccosus and parasitism of Arrhenophagus sp. and Pteroptrix sp. in P. aspidistrae, they are occurrence new record for do Rio de Janeiro State.

Keywords: Biological control; citrus; Hymenoptera; natural enemies; parasitism.

A s cochonilhas são insetos fitófagos, parasitas de plantas que, em grandes populações, prejudicam seus hospedeiros. Em ecossistemas equilibrados elas são biologicamente controladas por seus inimigos naturais (SiLva et al. 2007).

O levantamento das espécies de inimigos naturais que atuam no controle das cochonilhas de citros é de extrema importância, pois adequadamente manejados, podem manter as populações de insetos prejudiciais em densidades abaixo do nível de dano econômico (WolfF et al. 2004).

O aleirodídeo Aleurothrixus floccosus (Maskell) em São Paulo, é parasitado por três espécies de microhimenópteros, sendo eles: Eretmocerus paulistus Hempel, Prospatella brasiliensis (Hempel) e Signiphora towsendi Ashmead (Fonseca 1934; RANGEL \& Gomes 1945). Na República Argentina, os parasitóides primários de A.floccosus, são Cales noacki Howard, Signiphora xanthographa Blanchard e Amitus spiniferus (Brèthes) (DE SANTIS 1941) citado por Costa Lima (1942).

Os aleirodídeos estão amplamente distribuídos geograficamente e vivem sobre um grande número de plantas silvestres e ornamentais. Entretanto, diversas espécies tem sido apontadas como pragas de culturas de importância econômica, por simplesmente sugarem a seiva das plantas, outras por transmitirem viroses e substâncias toxicogênicas, por facilitarem o ataque de patógenos ou proporcionarem o aparecimento de fumagina sobre seus dejetos (CASsino \& NASCIMENTO 1999).

No Brasil, segundo Costa Lima (1942), A.floccosusé eficientemente controlado pelos Calcidóideos E. paulistus, $P$. brasiliensis e $S$. towsendi; nas Antilhas, além destas espécies, ainda apresentam-se como parasitoides, eficientes espécies de Encarsia (Aphelinidae) e o Entodotídeo, Euderomphale aleurothrixi Dozier. De acordo 
com Costa Lima (1962) A. floccosus é parasitado por C. noacki, $E$. paulistus, $P$. brasiliensis, S. townsendi, S. xanthographa. Ainda no Brasil A. floccosus é parasitado por E. paulistus (SP), $P$. brasiliensis (SP) e $S$. townsendi (SP e RJ), já Pinnaspis spp. é parasitado por Aphytis lingnanensis Compere (DE SANTIS 1980).

Informações de Nascimento et al. (1982), indicam que as espécies A. lignanensis e Aphytis. holoxanthus De Bach foram introduzidas, no Brasil em 1962, com a finalidade de controlar os diaspidídeos conhecidos vulgarmente por "escama-vírgula", "escama-farinha" e "escama-cabeça de prego".

De acordo com MiKLASIEWicz \& WALKER (1990), os maiores inimigos naturais de $A$. floccosus, encontrados em seus estudos foram C. noacki e Eretmocerus sp. Em estudos sobre eficiência do controle biológico de aleirodídeos em citros, BARBAGALLO et al (1992) observaram que $A$. floccosus foi controlado eficientemente por dois parasitóides, C. noacki e Encarsia meritoria Gahan.

Dentre os aleirodídeos Dialeurodes citri (Ashmead), Parabemisia myricae (Kuwana), A. floccosus e Paraleyrodes minei Iaccarino, Ulusoy et al. (1996), citaram que somente $P$. myricae é controlado pelo parasitóide Eretmocerus debachi (Rose \& Rosen). Os autores afirmaram que o controle com óleo mineral é mais eficiente para $D$. citri e que para $A$. floccosus e $P$. minei, estão sendo desenvolvidos métodos de controle, pois são duas espécies recém introduzidas, no Mediterrâneo Oriental, na região da Turquia. UyGUn et al. (1997) citaram que as pragas mais sérias dos citros são as moscas brancas $D$. citri, $P$. myricae, $A$. floccosus e P. minei.

A redução da população de $A$. floccosus em Atenas, segundo KATSOYANNOS et al. (1997) foi principalmente atribuída a introdução de $C$. noacki. Ainda sobre estudos de liberação inundativa de parasitóides na Grécia, Katsoyannos et al. (1998), verificaram que antes da liberação de $C$. noacki a densidade populacional de $A$. floccosus, era de 4,6-4,7 ninfas/superfície foliar (maior densidade) e nove meses depois a introdução do parasitóide o parasitismo obteve uma razão de $82,2 \%$.

Em estudos da importância econômica e de inimigos naturais de aleirodídeos, VISCARRET et al. (2000), verificaram Bemisia tabaci (Gennadius) complexo, Trialeurodes vaporiorium (Westwood), A. floccosus, Aleurothrixus aepim (Goeldi), D. citri, sendo parasitados pelos afelinídeos, Encarsia formosa Gahan, Encarsia lycopersicii De Santis, Encarsia porteri (Mercet), Encarsia. protansvena Viggiani, Encarsia transvena (Timberlake), Encarsia pergandiella Howard (grupo), Encarsia hispida De Santis, Encarisa sp., Eretmocerus corni Haldeman, Eretmocerus sp. e C. noacki, sendo ainda verificado o registro de nova ocorrência de E. protansvena, E. transvena para a Argentina.

O Objetivo do presente estudo foi registrar a ocorrência de parasitóides de aleirodídeos e cochonilhas associados à plantas cítricas no Estado do Rio de Janeiro.

\section{MATERIAL E MÉTODOS}

Foram visitados 12 municípios no Estado do Rio de Janeiro (Seropédica, Piraí, Itaboraí, Tanguá, Rio Bonito, Silva Jardim, Araruama, Iguaba Grande, São Pedro d'Aldeia, Cabo Frio, Saquarema e Maricá) entre dezembro de 2000 e junho de 2001, onde foram coletadas folhas com a presença dos seguintes fitoparasitos:

1. Aleirodídeos (Sternorrhyncha, Aleyrodidae): A. floccosus, Tetraleurodes (=Aleurotrachelus) cruzi (Cassino) e Paraleyrodes bondari Peracchi.

2. Cochonilhas: Coccus viridis ficus (Ashmead), Pinnaspis (Sternorrhyncha, Diaspididae)

(Green), Crysomphalus aspidistrae (Signoret) e Orthezia praelonga
(Douglas) (Sternorrhyncha, Ortheziidae).

As folhas foram coletadas e acondicionadas em sacos de papel ( $2 \mathrm{Kg}$ ), devidamente identificados com: o nome do fitoparasito, data de coleta, espécie vegetal e município de procedência. Ao serem levadas ao laboratório, estas folhas passaram por um procedimento de triagem que consistia em:

1. Verificação de vida do fitoparasito, baseando-se no estado físico do mesmo;

2. Limpeza da folha;

3. Eliminação dos demais organismos encontrados na folhas, diferentes ao objeto de estudo; cada folha permanecia com uma única espécie de fitoparasitos, para que o parasitóides que surgissem fossem mesmo associados àquela espécie;

4. Incubação das folhas em placas de Petri, devidamente identificadas com os mesmos dados contidos no saco de papel, além de receberem um código específico, que identificava a cidade de procedência, fitoparasito estudado, número de ordem e espécie vegetal.

Operíodo de incubação foi padronizado para 25 dias. Olaboratório possuía ambiente controlado, sendo a temperatura $25 \pm 2{ }^{\circ} \mathrm{C}$, Umidade Relativa do $\operatorname{Ar} 75 \pm 5 \%$ e fotofase de 14 horas.

Após o período de incubação as placas passaram pela seguinte triagem:

1. Separação dos microhimenópteros por morfo-espécie;

2. Acondicionamento das morfo-espécies em tubos de plásticos contendo álcool $70 \%{ }^{\circ} \mathrm{GL}$, previamente preparados;

3. Etiquetagem dos tubos com o código referente à placa de Petri.

Os dados referentes ao material coletado, incubado e triado, foram anotados em caderno laboratorial específico, para um maior controle.

\section{RESULTADOS E DISCUSSÃO}

Foram identificados 10 parasitóides associados aos fitoparasitos, sendo que nove destes identificados em nível de gênero e um em nível de família (Platygasteridae).

No período de estudo, somente A. floccosus e P. aspidistrae foram expressivamente parasitados (Tabela 1), já os demais fitoparasitos estudados, não foi observado parasitismo, com exceção de $T$. cruzi, que foi obtido um espécime de parasitóide e de $C$. viridis, dois espécimes de um mesmo parasitóide. Devido ao número reduzido de espécimes obtidos no parasitismo deste dois últimos homópteros, não foi possível envia-los para identificação.

Entre $P$. aspidistrae e A. floccosus, o aleirodídeo foi onde houve um maior número de espécies de parasitóides, oito dos dez encontrados (Arrhenophagus sp., Cales sp., Encarsia sp.(1), Eretmocerus sp., Signiphora sp.(1), Signiphora sp.(2), Signiphora sp.(3) e um parasitóide da família Plastygasteridae), Duas espécie foram observadas parasitando tanto o aleirodídeo quanto a cochonilha (Cales sp. e Arrhenophagus sp), verificandose uma menor especificidade em relação aos demais parasitóides observados.

A cochonilha Pinnaspis é parasitada por A lignanesis, Encarsia e Pteroptrix chinensis (Howard), na Índia. E a espécie Pinnaspis strachani (Cooley) é parasitada por Arrhenophagus chionaspidis Aurivillus, entre outros (FRY 1987), entretanto o presente estudo verificou somente o parasitismo da cochonilha por Arrhenophagus, Cales sp., Encarsia sp.(2) e Pteroptrix sp, para $P$. aspidistrae. 
Tabela 1. Parasitóides associados a A. floccosus, P. aspidistrae, T. cruzi e C. viridis (Homoptera: Sternorrhyncha) em diversas espécies de citros, coletados em 12 municípios do Estado do Rio de Janeiro, no período de dezembro de 2000 a junho de 2001.

\begin{tabular}{|c|c|c|c|c|}
\hline \multirow{2}{*}{ Parasitóide } & \multicolumn{4}{|c|}{ Homóptero } \\
\hline & A. floccosus & P. aspidistrae & T. cruzi & C. viridis \\
\hline Arrhenophagus sp. & $\mathrm{X}$ & $\mathrm{X}$ & & \\
\hline Cales sp. & $\mathrm{X}$ & $\mathrm{X}$ & & \\
\hline Encarsia sp. (1) & $\mathrm{X}$ & & & \\
\hline Encarsia sp. (2) & & $\mathrm{X}$ & & \\
\hline Eretmocerus sp. & $\mathrm{X}$ & & & \\
\hline Plastygasteridae & $\mathrm{X}$ & & & \\
\hline Pteroptrix sp. & & $\mathrm{X}$ & & \\
\hline Signiphora sp. (1) & $\mathrm{X}$ & & & \\
\hline Signiphora sp. (2) & $\mathrm{X}$ & & & \\
\hline Signiphora sp. (3) & $\mathrm{X}$ & & & \\
\hline sp. não identificada 1 & & & $\mathrm{X}$ & \\
\hline sp. não identificada 2 & & & & $\mathrm{X}$ \\
\hline$\Sigma$ & 8 & 4 & $\mathbf{1}$ & 1 \\
\hline
\end{tabular}

Mesmo existindo citações na literatura do parasitismo de espécies do gênero Aphytis associados à diaspidídeos (no presente estudo, $S$. articulatus, $C$. ficus, $P$. aspidistrae e $M$. beckii), este parasitóides não foram observadas, apesar de AzEvEDo (1996), verificarem o parasitismo de S. articulatus por Aphytis spp. na Região citrícola do Estado do Rio de Janeiro.

No Rio Grande do Sul, Wollf et al. (2004) verificaram que os diaspidídeos encontrados no estudo são potencialmente pragas, porém estão presentes também os organismos que controlam naturalmente a população destes insetos, tais como: parasitóides (Hymenoptera, Chalcidoidea), predadores (Coccinelidae e Chrysopidae) e fungos entomopatogênicos, o que reforça a importância de minimizar a utilização de produtos fitossanitários que podem interferir no equilíbrio destas populações.

Quando considerando o parasitismo em aleirodídeos estudos anteriores (RoDrigues \& CASSINO; 200; RodRIGUES \& CASSINO 2004) já havia registro de A. floccosus parasitado por Encarsia sp. e Signiphora sp., no Rio de Janeiro.

CASsino \& Nascimento (1999) citaram que não haviam verificado a predação/parasitismo de T. cruzi. Apesar da não identificação da espécie ou mesmo o gênero do parasitóide associado a este aleirodídeo, este é o primeiro registro da ocorrência de parasitismo nesta espécie de aleirodídeo, para o Estado do Rio de Janeiro, podendo ser a primeira citação para o mundo.

Além de Trialeurodes variabilis (Quaintance), E. hispida já foi encontrada parasitando 24 espécies de Aleyrodidae, pertencentes aos gêneros Aleurodicus, Aleuroglandulus, Aleurothrixus, Aleurotrachelus, Aleurotuba, Aleyrodes, Bemisia, Crenidorsum, Lecanoideus, Lipaleyrodes, Parabemisia, Siphoninus, Tetraleurodes e Trialeurodes em 18 países das Américas e Europa (HERnÁndEZ-SuÁrez et al. 2003; NoYES 2011).

Com poucos estudos realizados sobre parasitóides de fitoparasitos de plantas cítricas no Estado do Rio de Janeiro, verificaramse novos registros de ocorrência de parasitismo, como é o caso de Arrhenophagus sp. e Pteroptrix sp. em P. aspidistrae; e de Arrhenophagus sp. e do parasitóide da família Platygasteridae em $A$. floccosus. Deste modo, estudos mais aprofundados poderão ser realizados para entendimento da biologia e potencial de parasitismo destes himenópteros.

\section{AGRADECIMENTOS}

Aos pesquisadores Dra. Maria Angélica Penteado Dias (Universidade Federal de São Carlos) e o Dr. Valmir Antônio Costa (Instituto Biológico de Campinas), pela gentil identificação dos parasitóides.

\section{REFERÊNCIAS}

Azevedo, O.R.F., 1996. Diagnóstico da Citricultura no Estado do Rio de Janeiro. Dissertação (Mestrado em Fitotecnia),
Universidade Federal Rural do Rio de Janeiro, Itaguaí, RJ, $164 \mathrm{p}$.

Barbagallo, S., S. Longo, I. Patti \& C. Rapisarda, 1992. Efficiency of biological control against citrus whiteflies in Italy, Bolletino Zoologia Agraria Bachiocultura 24: 121-135.

Cassino, P.C.R. \& F.N. Nascimento, 1999. Aleirodídeos (Homoptera, Aleyrodidae) em plantas cítricas no Brasil: Distribuição e identificação. Anais da Sociedade de Entomologia do Brasil, 28: 75-83.

Costa Lima, A. M., 1942. Insetos do Brasil, $3^{0}$ Tomo, Homópteros. Esc. Nac. de Agronomia, Série Didática, n. ${ }^{\circ} 4$, Rio de Janeiro, $327 \mathrm{p}$.

Costa Lima, A.M., 1962. Insetos do Brasil, $12^{\circ}$ Tomo, Himenópteros. Esc. Nac. Agronomia, Série Didática, n. ${ }^{0} 14$, Rio de Janeiro, 393p.

De Santis, L., 1980. Catalogo de los himenopteros Brasileños de la serie parasitica - Yncluyendo Bethyloidea. Ed. Univ. Fed. Paraná, 395p.

Fonseca, J.P. da., 1934. Relação das principais pragas observadas nos anos de 1931, 32 e 33, nas plantas de maior cultivo no Estado de São Paulo, Arquivos do Instituto Biológico de São Paulo, 5: 263-289.

Fry, J.M., 1987. Natural Enemies Databank. A catalogue of natural enemies of arthropods derived from records in the CAB Natural Enemies Databank. CAB Intern. Int. Biol. Control, 185p.

Hernández-Suárez, E., A. Carnero, A. Aguiar, G. Prinsloo, J. LaSalle \& A. Polaszek. 2003. Parasitoids of whiteflies (Hymenoptera: Aphelinidae, Eulophidae, Platygastridae; Hemiptera: Aleyrodidae) from the Macaronesian archipelagos of the Canary Islands, Madeira and the Azores. Systematics and Biodiversity, 1: 55-108.

Katsoyannos, P., D.C. Kontodimas, \& G.J. Stathas, 1998. The inundative release of Cales noacki Howard (Hymenoptera, Aphelinidae), for curativetreatment of Aleurothrixusfloccosus (Maskell) (Homoptera: Aleyrodidae) on heavily infested citrus in Greece. Annales de l'Institut Phytopathologique Benaki, 18: 111-122.

Katsoyannos, P., K. Ifantis \& D.C. Kontodimas, 1997. Phenology, population trend and natural enemies of Aleurothrixus floccosus (Hom.: Aleyrodidae) at a newly invaded area in Athens, Greece. Entomophaga, 42: 619-628.

Miklasiewicz, T.J. \& G.P. Walker. 1990. Population dynamics and biological control of the woolly whitefly (Homoptera: Aleyrodidae) on citrus. Enviormental Entomology, 19: 14851490.

Nascimento, A.S., G.J. Morais, J.R.M Cabrita, L.M.S Silva, O.M. Porto, P.C.R. Cassino, S. Gravena \& W.B.S. Pinto. 1982. Manual de Manejo Integrado de Pragas do Pomar Cítrico. Brasília: EMBRAPA-DID, 48p.

Noyes, J.S. 2011. Universal Chalcidoidea Database. World Wide Web electronic publication. Disponível em: $<$ http://www.nhm.ac.uk/chalcidoids $>$. [Setembro de 2011].

Rangel, J. F. \& J.G. Gomes. 1945. Guia para reconhecimento e combate das principais doenças e pragas da laranjeira. 
Serviço de Documentação do Min. Agricultura, n. ${ }^{\circ}$ 11, Rio de Janeiro, 47p.

Rodrigues, W.C. \& P.C.R. Cassino. 2004. Inimigos Naturais, p. 97-114. In: P.C.R. Cassino \& W.C. Rodrigues (cords.), Citricultura Fluminense: Principais pragas e seus inimigos naturais. Seropédica, EDUR, 168p.

Rodrigues, W.C. \& P.C.R., Cassino. 2003. Parasitismo de Aleurothrixus floccosus (Homoptera, Aleyrodidae) por Encarsia sp. (Hymenoptera, Aphelinidae) e Signiphora sp. (Hymenoptera, Signiphoridae) em Tangerina (Citrus reticulata) cv. Poncã. Revista da Universidade Rural, Série Ciência da Vida, 23: 31-37.

Silva, L.N., V.R.S. Wolff, C.E. Pulz \& D.C. Silva, 2007. Predadores e parasitóides de diaspididae (Hemiptera; Sternorrhyncha) em citros - coleção didática do museu Prof. Ramiro Gomes Costa. Revista Brasileira de Agroecologia, 2: 745-748.

Ulusoy, M.R., N. Uygun, U. Kersting, I. Karaca \& S. Satar, 1996. Present of citrus whiteflies (Homoptera: Aleyrodidae) in Turkey and their control. Journal of Plant Diseases and Protection, 103: 397-402.
Uygun, N., M.R. Ulusoy, I. Karaca, U. Kersting \& V. Vacanta, 1997. Approaches to biological control of Dialeurodes citri (Ashmead) in Turkey. Bulletin OILB SROP, 20: 52-62.

Viscarret, M.M., E.N Botto \& A. Polaszeck, 2000. Whiteflies (Hemiptera: Aleyrodidae) of economic importance and their natural enemies (Hymenoptera: Aphelinidae, Signiphoridae) in Argentina. Revista Chilena Entomologia, 26: 5-11.

Wolff, V.R.S., C.E. Pulz, D.C. Silva, J.B. Mezzomo \& C.A. Prade. 2004. Inimigos naturais associados à diaspididae (Hemiptera, Sternorrhyncha), ocorrentes em Citrus sinensis (Linnaeus) Osbeck, no Rio Grande do Sul, Brasil: I - joaninhas e fungos entomopatogênicos. Arquivos do Instituto Biológico de São Paulo, 71: 355-361.

Recebido em: 17/o7/2011

Aceito em: 14/10/2011

Como citar este artigo:

Rodrigues, W.C. \& P.C.R. Cassino, 2012. Parasitóides Associados a Cochonilhas e Aleirodídeos (Sternorrhyncha) de Plantas Cítricas no Estado do Rio de Janeiro. EntomoBrasilis, 5(1): 33-36.

Acessível em: http://www.periodico.ebras.bio.br/ojs/index.php/ebras/article/view/177
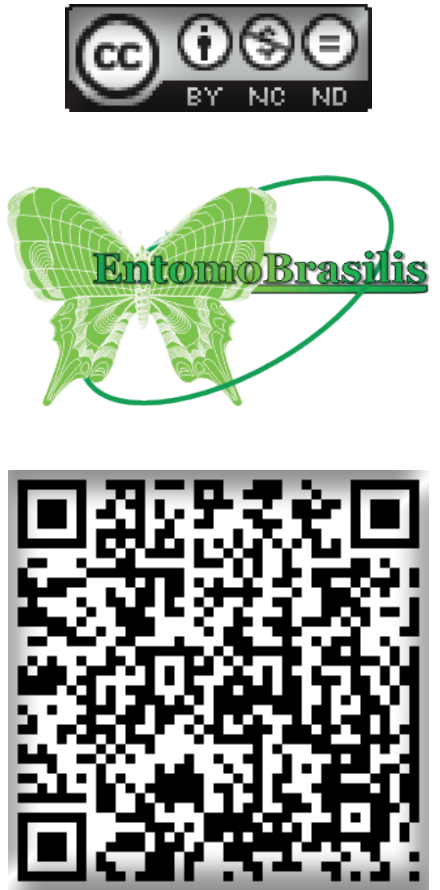